01

\title{
Расчет химических сдвигов рентгеновских эмиссионных спектров ниобия в оксидах ниобия(V) относительно металла*
}

\author{
( Ю.В. Ломачук ${ }^{1}$, Ю.А. Демидов ${ }^{1}$, Л.В. Скрипников ${ }^{1,2}$, А.В. Зайцевский ${ }^{1,3}$, \\ С.Г. Семенов ${ }^{1}$, Н.С. Мосягин ${ }^{1}$, А.В. Титов ${ }^{1}$ \\ ${ }^{1}$ НИЦ Курчатовский институт - ПИЯФ, \\ 188300 Гатчина, Россия \\ ${ }^{2}$ Санкт-Петербургский государственный университет, фризический фракультет, \\ 198904 Санкт-Петербург, Россия \\ ${ }^{3}$ Московский государственный университет, химический факультет, \\ 119991 Москва, Россия \\ e-mail: lomachuk_yv@pnpi.nrcki.ru
}

Поступила в редакцию 28.11.2017 г.

Рассчитаны химические сдвиги линий $K_{\alpha 1}, K_{\beta 1}$ рентгеновских эмиссионных спектров ниобия в оксидах $\left(\mathrm{Nb}_{2} \mathrm{O}_{5}\right)_{n}, n=1-4$, относительно металлического $\mathrm{Nb}$. Стехиометрические кластеры $\left(\mathrm{Nb}_{2} \mathrm{O}_{5}\right)_{n}$, электронная структура которых была рассчитана с использованием прецизионных релятивистских псевдопотенциалов и двухкомпонентного варианта теории функционала плотности, рассматриваются как прообразы для моделирования различных кристаллических форм оксида ниобия $(\mathrm{V})$. Химические сдвиги вычислялись при помощи метода, основанного на использовании свойства приближенной пропорциональности валентных спиноров в остовной области тяжелого атома. Определены поправки к значениям химических сдвигов, учитывающие отклонения от указанной пропорциональности. Продемонстрирована быстрая сходимость результатов по отношению к размеру кластера оксида ниобия.

DOI: $10.21883 /$ OS.2018.04.45743.275-17

\section{1. Введение}

Основным препятствием для решения проблемы утилизации радиоактивных отходов является трудность прогнозирования поведения долгоживущих радионуклидов (особенно, искусственных чрезвычайно радиотоксичных актиноидов: $\mathrm{Pu}, \mathrm{Am}, \mathrm{Np}, \mathrm{Cm}$ ), которые в принципе могут находиться в химически и радиационно нестойкой форме в метамиктных минералах [1] в течение длительного времени $\left(10^{4}\right.$ и более лет). Природные минералы, содержащие примеси радиоактивных элементов, можно рассматривать как естественные модели изолированных в матрицах высокоактивных отходов на разных стадиях радиационного разрушения. Поэтому исследование структуры и свойств таких материалов необходимо для создания новых форм керамических матриц. Оксид ниобия (V) является основой широкого класса метамиктных соединений, в состав которых входят в примесных количествах радионуклиды $\mathrm{Y}, \mathrm{Ni}, \mathrm{Ti}$, $\mathrm{Ca}, \mathrm{Ta}, \mathrm{U}, \mathrm{Th}[2]$.

Химическая стабильность метамиктных соединений связана со способностью примесных атомов встраиваться в кристаллическую структуру ниобатов, для определения которой необходимо проведение квантовомеханических расчетов и сопоставления их с экспериментальными данными. Одним из методов экспериментального определения состояния атома в соединении

* Совещание по прецизионной атомно-молекулярной спектроскопии, 13-14 ноября 2017г., ПИЯФ НИЦ „Курчатовский институт“, Гатчина, Россия. является рентгеновская эмиссионная спектроскопия [3]. По изменению положения спектральной линии характеристического излучения атома в различных соединениях (химическом сдвиге линии) можно судить о его состоянии окисления и других свойствах. В работах $[4,5]$ предложен метод расчета химических сдвигов рентгеновских эмиссионных линий на основе результатов квантово-механического моделирования соединений. В работе [6] был проведен расчет химических сдвигов ниобия в ферсмите $\left(\mathrm{CaNb}_{2} \mathrm{O}_{6}\right)$ относительно металла и проведено сравнение с экспериментальными данными (величина химического сдвига $K_{\alpha 1}$-линии ниобия в этом соединении около $260 \mathrm{meV}$, теоретическое значение около $300 \mathrm{meV})$.

Целью настоящей работы является оценка химических сдвигов рентгеновских эмиссионных линий ниобия в $\mathrm{Nb}_{2} \mathrm{O}_{5}$ относительно металла. Анализ погрешностей этих величин для структур, соответствующих реальным кристаллам оксида ниобия $(\mathrm{V})$, осложнен тем, что данное соединение имеет несколько кристаллических форм сравнительно сложного строения [7]. Поэтому нами был использован другой подход, заключающийся в моделировании кристалла молекулярными кластерами $\left(\mathrm{Nb}_{2} \mathrm{O}_{5}\right)_{n}, n=1-4$. Такие кластеры ранее изучались в работах $[8,9]$.

Расчет химических сдвигов линий $K_{\alpha 1}$ и $K_{\beta 1}$ ниобия в этих кластерах позволяет детально проанализировать точность предложенного метода $[4,5]$, а также изучить влияние размера рассматриваемых кластеров на величину химического сдвига. 


\section{2. Процедура расчета химических сдвигов рентгеновских эмиссионных спектров}

Моделирование структуры и свойств $\mathrm{Nb}_{2} \mathrm{O}_{5}$ и металлического ниобия выполнено в рамках кластерного приближения. Основой методики решения многоэлектронной задачи для кластеров послужили расчетные технологии релятивистской теории функционала плотности в ее „двухкомпонентной“ неколлинеарной формулировке [10], адаптированные к использованию модели прецизионных „согласованных по форме“ релятивистских псевдопотенциалов малых остовов [11-13]. В рамках этой модели, в отличие от случая явного описания всех электронных оболочек рассматриваемых систем, вполне корректно использование формально нерелятивистской аппроксиманты PBE0 [14] обменно-корреляционного функционала. Релятивистские двухкомпонентные уравнения Кона-Шема решались посредством разложения компонент вспомогательных спиноров по базисным системам, оптимизированным с учетом магнитных (эффективных спин-орбитальных) взаимодействий. Особенности примененной технологии построения базисов, обеспечивающие хорошее соотношение „число базисных функций/ точность результатов“ - одновременная оптимизация нелинейных и линейных параметров сгруппированных гауссовых функций и возможность включения в процедуру оптимизации разнородных объектов, содержащих данный атом, что обеспечивает универсальность получаемых базисов. Базис для кислорода был получен расширением трехэкспонентного набора [15] поляризационными и диффузными функциями.

Двухэтапную процедуру, примененную в данной работе, можно считать обобщением описанной в работах [16-18]. На первом этапе выполняется молекулярный расчет в приближении релятивистского псевдопотенциала остова [11-13]. Псевдофункции, описывающие состояния валентных и внешних остовных электронов, „сглажены“ (модифицированы по сравнению с истинными одноэлектронными функциями, имеющими большое число радиальных узлов) в остовной области атома. Для расчета ряда свойств, обусловленных поведением волновых функций вблизи ядра, требуется восстановить правильное поведение четырехкомпонентных аналогов псевдофункций (биспиноров) в этой области. Для этого на втором этапе строится пара одноцентровых эквивалентных базисных наборов. В первый включаются атомные псевдофункции. Во второй набор включаются четырехкомпонентные функции, рассчитанные с гамильтонианом Дирака-Кулона(Брейта) для тех же конфигураций атома, что и функции из первого набора. Далее молекулярные псевдофункции (псевдоспиноры) переразлагаются по одноцентровым атомным псевдофункциям, коэффициенты переразложения $c_{n l j m}^{i}$ фиксируются и атомные псевдофункции заменяются соответствующими эквивалентными атомными биспинорами. В результате получается следующее выражение для молекулярного спинора $\varphi_{i}(\mathbf{r})$ :

$$
\varphi_{i}(\mathbf{r})=\sum_{n l j m} c_{n l j m}^{i}\left(\begin{array}{c}
f_{n l j}(r) \Omega_{l j m}(\mathbf{r} / r) \\
g_{n l j}(r) \Omega_{2 j-l, j m}(\mathbf{r} / r)
\end{array}\right) .
$$

В этом выражении $f_{n l j}(r)$ и $g_{n l j}(r)$ - радиальные функции большой и малой компоненты атомного биспинора, соответствующего значениям главного квантового числа $n$, орбитального и полного моментов $l$ и $j$; $\Omega_{l j m}(\mathbf{r} / r)$ - двухкомпонентные сферические спиноры, которые являются собственными функциями операторов полного момента количества движения и пространственной инверсии.

Построив одночастичную молекулярную матрицу плотности $\rho$ в базисе атомных биспиноров и просуммировав по $n$, можно определить распределение электронов по „парциальным волнам“ относительно ядра данного атома.

Полученное представление матрицы плотности было использовано для расчета постоянных сверхтонкой структуры и других свойств атома в соединении с погрешностью 5-10\% в работах [19-22].

Воспользуемся свойством приближенной пропорциональности валентных (и низколежащих виртуальных в случае корреляционного расчета) спиноров в остовной области рассматриваемого атома [11]. Тогда переразложение можно выполнять не во всем пространстве, а только внутри сферы с центром на ядре рассматриваемого атома с радиусом $R_{c}$. В этом случае разложение (1) приобретает следующий вид:

$$
\varphi_{i}\left(\mathbf{r}<R_{c}\right)=\sum_{l j m} c_{l j m}^{i}\left(\begin{array}{c}
\eta_{l j}^{f}(r) \Omega_{l j m}(\mathbf{r} / r) \\
\eta_{l j}^{g}(r) \Omega_{2 j-l, j m}(\mathbf{r} / r)
\end{array}\right), r<R_{c},
$$

где $\eta_{l j}^{f}(r)$ и $\eta_{l j}^{g}(r)$ - радиальные части большой и малой компонент некоторых реперных биспиноров, которые заранее выбираются для каждой пары значений $l$ и $j$. Нормировка $\eta_{l j}(r)=\left(\begin{array}{c}\eta_{l j}^{f}(r) \\ \eta_{l j}^{g}(r)\end{array}\right)$ определяется соотноше-
нием

$$
\int_{r<R_{c}}\left|\eta_{l j}(r)\right|^{2} r^{2} d r=1 .
$$

Величину любого свойства $A$ атома в соединении, описываемого одноэлектронным оператором $\mathbf{A}$, локализованным в остовной области рассматриваемого атома, можно получить, вычислив среднее значение этого оператора с использованием матрицы плотности $\boldsymbol{\rho}_{r<R_{c}}$, записанной в представлении (2):

$$
A=\operatorname{Tr}\left[\boldsymbol{\rho}_{r<R_{c}} \mathbf{A}\right] .
$$

Описываемая процедура была реализована и использована для количественного объяснения соотношения вычисленных постоянных сверхтонкой структуры и других свойств, зависящих от спиновой электронной плотности, 
в молекулах $\mathrm{ThO}$ и $\mathrm{ThF}^{+}$[18]. Однако для расчета химических сдвигов рентгеновских эмиссионных спектров ее точность может быть недостаточной, поскольку эти величины представляют собой разности близких по величине средних значений эффективного одноэлектронного оператора $\chi[4]$, вычисленных по матрицам плотности, описывающим электронную структуру соответствующих соединений.

Естественным обобщением указанного метода может быть использование нескольких реперных биспиноров для каждой пары $l$ и $j$. Для оценки погрешности, связанной с нарушением свойства пропорциональности, в настоящей работе рассматривается случай двух реперных биспиноров для каждой пары значений $l$ и $j$. Обозначим радиальные части больших и малых компонент этих биспиноров как $\eta^{1, f}(r), \eta^{1, g}(r)$ и $\eta^{2, f}(r), \eta^{2, g}(r)$ для первого и второго биспинора соответственно.

Выражение (2) принимает следующий вид:

$$
\begin{gathered}
\varphi_{i}\left(\mathbf{r}<R_{c}\right) \\
=\sum_{l j m}\left(\begin{array}{c}
\left(c_{l j m}^{i, 1} \eta^{1, f}(r)+c_{l j m}^{i, 2} \eta^{2, f}(r)\right) \Omega_{l j m}(\mathbf{r} / r) \\
\left(c_{l j m}^{i, 1} \eta^{1, g}(r)+c_{l j m}^{i, 2} \eta^{2, g}(r)\right) \Omega_{2 j-l, j m}(\mathbf{r} / r)
\end{array}\right), \\
r<R_{c} .
\end{gathered}
$$

Условие ортонормировки (3) в этом случае может быть записано как

$$
\int_{r<R_{c}} \eta_{l j}^{p}(r) \eta_{l j}^{q}(r) r^{2} d r=\delta_{p q} .
$$

Радиальные части реперных биспиноров в настоящей работе получены взаимной ортогонализацией биспиноров, соответствующих одноэлектронным валентным и низколежащим виртуальным состояниям, взятым из расчета методом Дирака-ХартриФока свободного атома $\mathrm{Nb}$ в конфигурации $1 s^{2} 2 s^{2} 2 p^{6} 3 s^{2} 3 p^{6} 3 d^{10} 4 s^{2} 4 p^{6} 4 d^{4} 5 s^{1} \quad$ с использованием программы HFD [23].

Реперные биспиноры, соответствующие парциальной волне $s_{1 / 2}$, были получены ортогонализацией по условию (6) из биспиноров $5 s$ и $6 s$; парциальным волнам $p_{1 / 2}$ и $p_{3 / 2}$ - из соответствующих биспиноров $4 p_{1 / 2}$, $5 p_{1 / 2}$ и $4 p_{3 / 2}, 5 p_{3 / 2}$; парциальным волнам $d_{3 / 2}$ и $d_{5 / 2}-$ из биспиноров $4 d_{3 / 2}, 5 d_{3 / 2}$ и $4 d_{5 / 2}, 5 d_{5 / 2}$.

\section{3. Результаты расчетов}

Для оценки применимости свойства пропорциональности при вычислении химических сдвигов линий $K_{\alpha 1}$ (переход $2 p_{3 / 2} \rightarrow 1 s_{1 / 2}$ ), $K_{\beta 1} \quad$ (переход $3 p_{3 / 2} \rightarrow 1 s_{1 / 2}$ ) атома $\mathrm{Nb}$, проведены расчеты иона $\mathrm{Nb}^{+3}$ и нейтрального атома в приближении Дирака-Хартри-Фока-Брейта с использованием программы HFD [23]. Для случая свободных атомов и ионов при пренебрежении погрешностями, связанными с использованием процедуры восстановления электронной плотности в остовной области

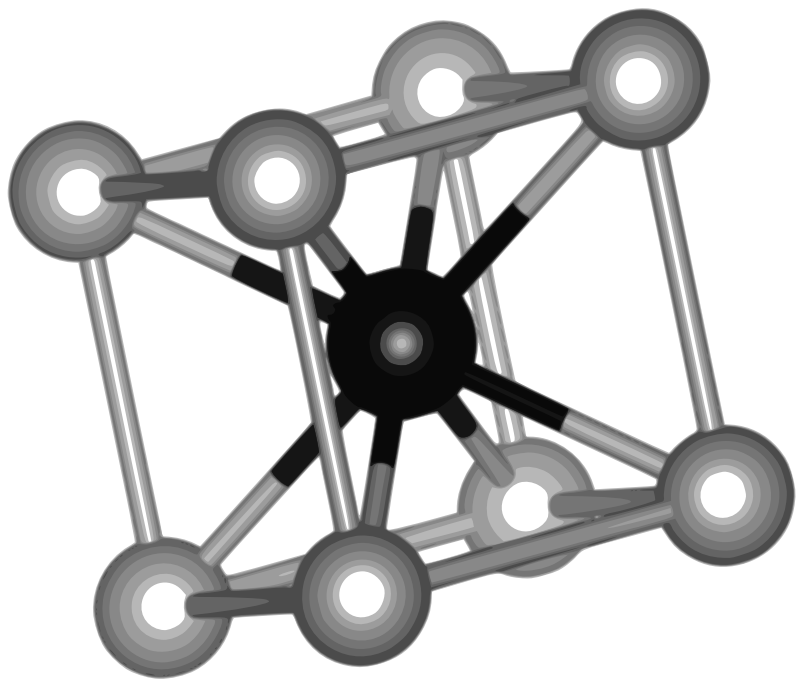

Рис. 1. Кластер $\mathrm{Nb}_{9}$ состоит из центрального атома $\mathrm{Nb}$ (выделен черным цветом) и его первой координационной сферы в структуре ОЦК. Расстояния между соседними атомами ниобия в кластере совпадают с экспериментальным значением расстояния $\mathrm{Nb}-\mathrm{Nb}$ в идеальном кристалле $(2.86 \AA)$.

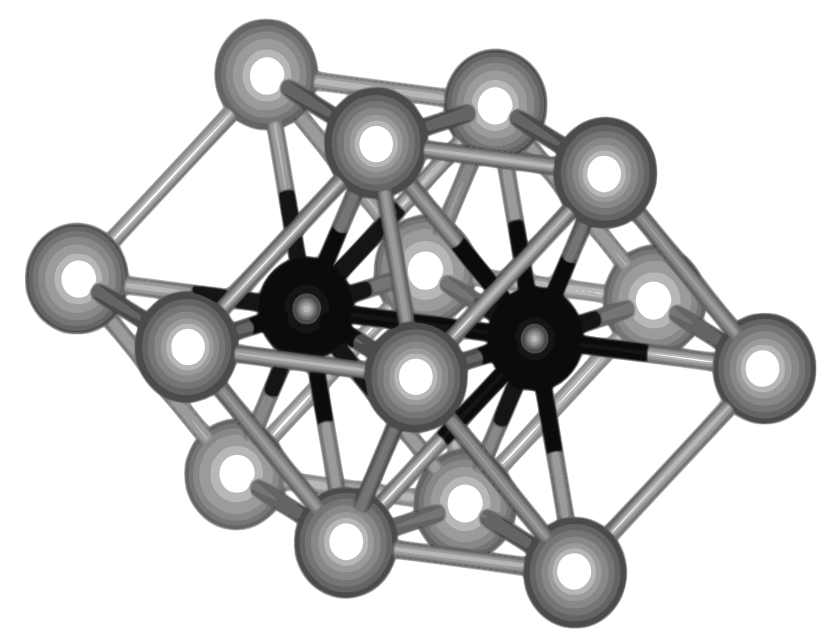

Рис. 2. Кластер $\mathrm{Nb}_{16}$ состоит из двух соседних („центральных“, на рисунке выделены черным цветом) атомов $\mathrm{Nb}$ и обьединения их первых координационных сфер. Расстояния между соседними атомами ниобия в кластере совпадают с экспериментальным значением расстояния $\mathrm{Nb}-\mathrm{Nb}$ в идеальном кристалле $(2.86 \AA)$.

атома [16,17], методика вычисления химических сдвигов рентгеновских эмиссионных спектров с использованием эффективного одночастичного оператора, описанная в работах $[4,5]$, должна приводить к результатам, совпадающим с оценками химических сдвигов как разности энергий соответствующего перехода в атоме и ионе, вычисленных по теореме Купманса [24].

Сравнивая эти величины, можно определить погрешность, вносимую при использовании различных вариантов процедуры восстановления. Из результатов, приве- 

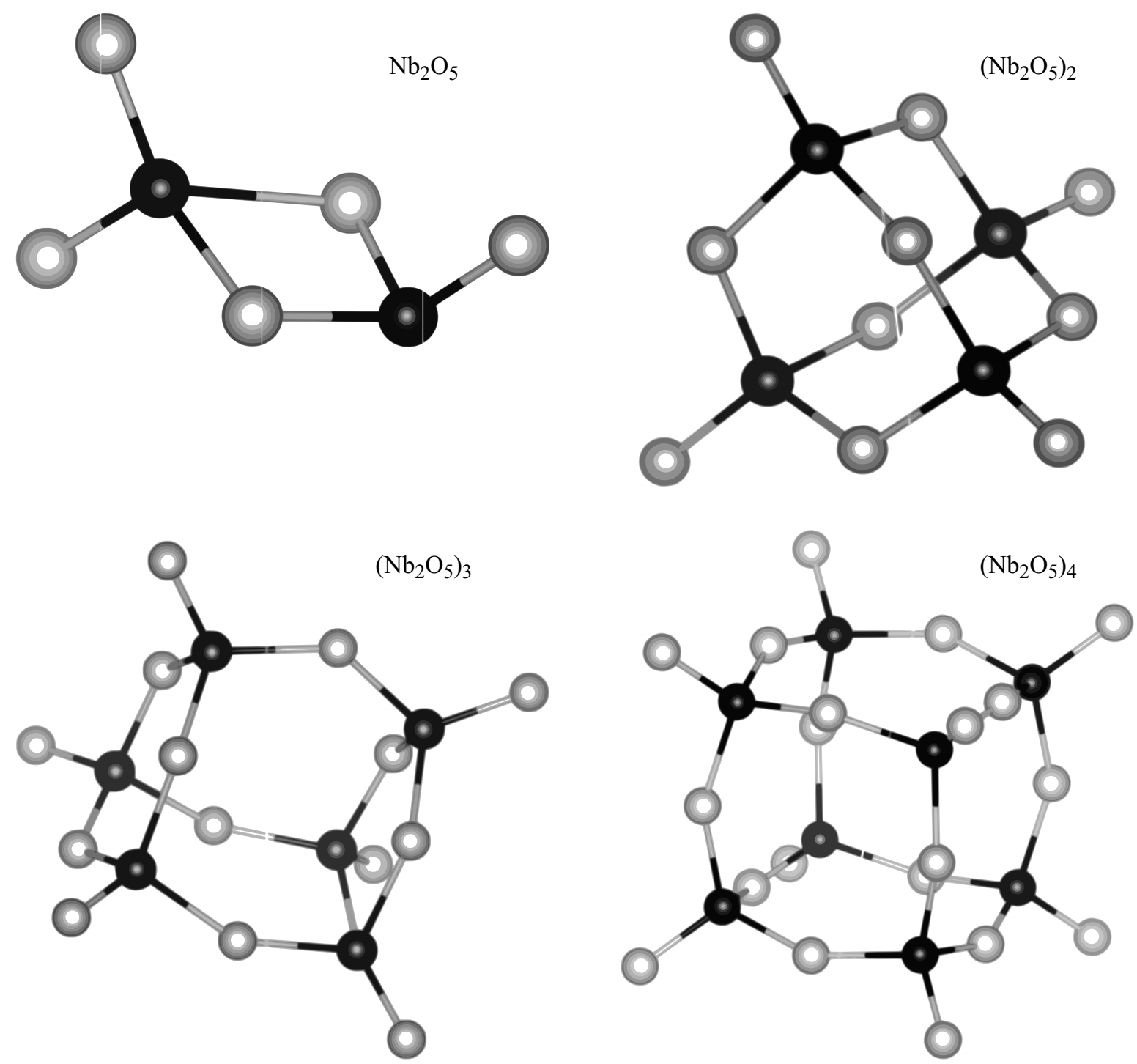

Рис. 3. Равновесные ядерные конфигурации молекулярных кластеров стехиометрического состава $\left(\mathrm{Nb}_{2} \mathrm{O}_{5}\right)_{n}$. Атомы ниобия выделены черным цветом.

денных в табл. 1, следует, что для вычисления химических сдвигов линии $K_{\alpha 1}$ с погрешностью около $20 \mathrm{meV}$ достаточно использования свойства пропорционально- сти валентных и внешнеостовных спиноров в остовной области атома. Для химических сдвигов линии $K_{\beta 1}$ погрешность составляет порядка $100 \mathrm{meV}$. Такая разница

Таблица 1. Химические сдвиги линий $K_{\alpha 1}$ (переход $2 p_{3 / 2} \rightarrow 1 s_{1 / 2}$ ) и $K_{\beta 1}$ (переход $3 p_{3 / 2} \rightarrow 1 s_{1 / 2}$ ) иона $\mathrm{Nb}^{+3}$ относительно нейтрального атома, $\mathrm{meV}$

\begin{tabular}{c|c|c|c|c}
\hline Переход & $R_{c}=0.5$ a. u. & $R_{c}=0.5$ a. u. $^{2}$ & $R_{c}=0.7$ a. u. ${ }^{2}$ & Теорема Купманса $^{3}$ \\
\hline $2 p_{3 / 2}-1 s_{1 / 2}$ & -200 & -219 & -216 & -220 \\
$3 p_{3 / 2}-1 s_{1 / 2}$ & 108 & 58 & 4 & 8
\end{tabular}

Примечание. Результаты, полученные с использованием:

1 свойства пропорциональности;

2 двух реперных биспиноров для каждой пары значений квантовых чисел $l$ и $j$;

3 оценки по теореме Купманса, соответствующие точному переразложению электронной плотности при рассмотрении свободного атома и иона. Расчеты проведены c помощью компьютерного кода [23] в приближении Дирака-Хартри-Фока. Конфигурация атома Nb $\left[1 s^{2}-3 d^{10}\right] 4 s^{2} 4 p^{6} 4 d^{4} 5 s^{1}$, изолированного иона $-\left[1 s^{2}-3 d^{10}\right] 4 s^{2} 4 p^{6} 4 d^{2}$. 
Таблица 2. Химические сдвиги линий $\mathrm{Nb} K_{\alpha 1}$ (переход $\left.2 p_{3 / 2} \rightarrow 1 s_{1 / 2}\right)$ и $K_{\beta 1} \quad\left(\right.$ переход $\left.3 p_{3 / 2} \rightarrow 1 s_{1 / 2}\right)$ в различных соединениях, моделирующих твердое тело, $\mathrm{meV}$

\begin{tabular}{c|c|c|c}
\hline Переход & $\mathrm{Nb}-\mathrm{Nb}_{9}{ }^{1}$ & $\mathrm{Nb}_{2}-\mathrm{Nb}_{9}{ }^{2}$ & $\mathrm{Nb}_{16}-\mathrm{Nb}_{9}{ }^{3}$ \\
\hline $2 p_{3 / 2} \rightarrow 1 s_{1 / 2}{ }^{4}$ & $120(90)$ & $120(100)^{5}$ & $-20(-14)$ \\
$3 p_{3 / 2} \rightarrow 1 s_{1 / 2}{ }^{5}$ & 340 & 430 & -9
\end{tabular}

Примечание. Химический сдвиг линий ниобия $K_{\alpha 1}$ и $K_{\beta 1}$ в:

1 свободном атоме $\mathrm{Nb}$ относительно кластера $\mathrm{Nb}_{9}$;

2 димере $\mathrm{Nb}_{2}$ относительно кластера $\mathrm{Nb}_{9}$;

3 кластере $\mathrm{Nb}_{16}$ относительно $\mathrm{Nb}_{9}$.

${ }^{4}$ Химические сдвиги линий ниобия $K_{\alpha 1}$ и $K_{\beta 1}$ рассчитаны с использованием двух реперных биспиноров для каждой пары значений квантовых чисел $l$ и $j$ для восстановления электронной плотности в области $r<R_{c}=0.7$ a.u.

${ }^{5}$ В скобках приведены значения химических сдвигов линии $K_{\alpha 1}$, полученные с использованием свойства пропорциональности валентных и внешнеостовных спиноров в остовной области атома $\mathrm{Nb}$ $r<R_{c}=0.55$ a.u.

Таблица 3. Длины связей, заряд ниобия по Бейдеру и химические сдвиги линий $\mathrm{Nb} K_{\alpha 1}$ и $K_{\beta 1}$ относительно $\mathrm{Nb}_{9}$ в кластерах $\left(\mathrm{Nb}_{2} \mathrm{O}_{5}\right)_{n}{ }^{1}$. Геометрия кластеров соответствует минимуму полной энергии

\begin{tabular}{l|c|c|c|c|c}
\hline Кластер & $\begin{array}{c}\mathrm{Nb}-\mathrm{O}, \\
\AA(\mathrm{I})\end{array}$ & $\begin{array}{c}\mathrm{Nb}-\mathrm{O}, \\
\AA(\mathrm{II})\end{array}$ & $\begin{array}{c}Q_{\mathrm{Nb}}{ }^{2}, \\
\text { a. u. }\end{array}$ & $\begin{array}{c}\chi_{K \alpha 1}{ }^{3}, \\
\mathrm{meV}\end{array}$ & $\begin{array}{c}\chi_{K \beta 1}{ }^{3}, \\
\mathrm{meV}\end{array}$ \\
\hline $\mathrm{Nb}_{2} \mathrm{O}_{5}$ & 1.714 & 2.117 & 2.49 & $-239(-217)$ & -152 \\
$\mathrm{Nb}_{4} \mathrm{O}_{10}$ & 1.695 & 1.928 & 2.67 & $-303(-268)$ & -161 \\
$\mathrm{Nb}_{6} \mathrm{O}_{15}$ & 1.692 & 1.925 & 2.68 & $-303(-266)$ & -166 \\
$\mathrm{Nb}_{8} \mathrm{O}_{20}$ & 1.691 & 1.912 & 2.69 & $-310(-268)$ & -182
\end{tabular}

Примечание. ${ }^{1}$ Атом кислорода (I) связан только с одним атомом $\mathrm{Nb}$, (II) - с двумя атомами $\mathrm{Nb}$ (рис. 3).

${ }^{2}$ Вычислены с помощью кода $[27,28]$

${ }^{3}$ Химические сдвиги линий $K_{\alpha 1}$ и $K_{\beta 1}$ рассчитаны с использованием двух реперных биспиноров для каждой пары значений $l$ и $j$ при одноцентровом переразложении, $R_{c}=0.7$ a.u. (в скобках приведены результаты для линии $K_{\alpha 1}$, полученные с использованием свойства пропорциональности в области $r<R_{c}=0.55$ а. u.).

в погрешности связана с тем, что одноэлектронный оператор химического сдвига [4] локализован в области, размер которой пропорционален среднему радиусу оболочки, с которой происходит переход (для оболочки $3 p_{3 / 2}$ эта величина в 5 раз больше, чем для $2 p_{3 / 2}$ ).

Для повышения уровня точности расчета химического сдвига линии $K_{\beta 1}$ необходим учет поправок, связанных с отклонением от пропорциональности. Использование двух реперных биспиноров для восстановления электронной плотности в остовной области атома $\mathrm{Nb}$ размером $r<R_{c}=0.7$ a. u. позволяет снизить погрешность до $4 \mathrm{meV}$.

Для моделирования металлического $\mathrm{Nb}$ были проведены расчеты изолированного атома $\mathrm{Nb}$, димера $\mathrm{Nb}_{2}$ и двух кластеров $\mathrm{Nb}_{9}$ и $\mathrm{Nb}_{16}$ (рис. 1,2 ). Для расчета свободного атома и димера $\mathrm{Nb}_{2}$ использован псевдопотенциал 28-электронного остова $\mathrm{Nb}$ [25] (тринадцать элек- тронов ниобия, $4 s^{2} 4 p^{6} 4 d^{4} 5 s^{1}$, рассматривались явно). Моделирование кластеров $\mathrm{Nb}_{9}$ и $\mathrm{Nb}_{16}$ выполнялось с набором псевдопотенциалов остова, включающим в себя упомянутую 13-электронную модель для центрального атома $\mathrm{Nb}$ в $\mathrm{Nb}_{9}$ и двух центральных атомов $\mathrm{Nb}$ в $\mathrm{Nb}_{16}$ в сочетании с одноэлектронной моделью (четыре $d$ электрона включены в остов) для периферийных атомов. Расстояние между атомами $\mathrm{Nb}$ в кластерах полагалось равным экспериментальному значению $2.86 \AA$ [26].

Результаты расчетов представлены в табл. 2. Исходя из приведенных данных можно заключить, что значение химического сдвига существенно зависит от ближайшего окружения атома (химические сдвиги в свободном атоме $\mathrm{Nb}$ и димере $\mathrm{Nb}_{2}$ относительно кластеров $\mathrm{Nb}_{9}$ и $\mathrm{Nb}_{16}$ порядка $100 \mathrm{meV})$, но при этом практически не зависит от более удаленных атомов (химические сдвиги в $\mathrm{Nb}_{16}$ относительно $\mathrm{Nb}_{9}$ составляют около $20 \mathrm{meV}$ ).

При расчете молекулярных стехиометрических кластеров $\left(\mathrm{Nb}_{2} \mathrm{O}_{5}\right)_{n}$ проводилась оптимизация конфигурации ядер до достижения минимальной полной энергии системы. Равновесная геометрия кластеров показана на рис. 3. Каждый атом $\mathrm{Nb}$ в кластерах $\mathrm{Nb}_{4} \mathrm{O}_{10}, \mathrm{Nb}_{6} \mathrm{O}_{15}$ и $\mathrm{Nb}_{8} \mathrm{O}_{20}$ окружен четырьмя атомами кислорода, один из которых связан только с этим атомом $\mathrm{Nb}$, а три других являются мостиковыми. В кластере $\mathrm{Nb}_{2} \mathrm{O}_{5}$ два атома кислорода связаны с двумя атомами $\mathrm{Nb}$ одновременно. Это обстоятельство приводит к существенному различию длин связей и бейдеровских зарядов (табл. 3).

Результаты расчетов приведены в табл. 3. Для атома $\mathrm{Nb}$ использовался 13-электронный эффективный потенциал остова [25]. Значения химических сдвигов линий $\mathrm{Nb} K_{\alpha 1}$ и $K_{\beta 1}$ в оксиде $\mathrm{Nb}_{2} \mathrm{O}_{5}$ относительно металлического ниобия, моделируемого кластером $\mathrm{Nb}_{9}$, быстро стабилизируются при росте размера кластера - значения этих величин для $\mathrm{Nb}_{2} \mathrm{O}_{5}$ относительно металла отличаются от таковых в $\mathrm{Nb}_{4} \mathrm{O}_{10}$ на величину около $60 \mathrm{meV}$, в то время как соответствующее различие для кластеров $\mathrm{Nb}_{4} \mathrm{O}_{10}, \mathrm{Nb}_{6} \mathrm{O}_{15}$ и $\mathrm{Nb}_{8} \mathrm{O}_{20}$ порядка $10 \mathrm{meV}$. Сильное различие результатов в случае $\mathrm{Nb}_{2} \mathrm{O}_{5}$ и в случае бо́льших кластеров обусловлено качественным отличием ближайших окружений атомов $\mathrm{Nb}$.

\section{Заключение}

При вычислении химических сдвигов рентгеновских эмиссионных спектров тяжелых атомов в соединении требуется детальный анализ погрешностей. Это обстоятельство обусловлено тем, что химический сдвиг представляет собой разность двух средних значений эффективного одноэлектронного оператора [4], близких по величине. Итоговая относительная погрешность определения химического сдвига становится существенно больше по сравнению с другими остовными свойствами атома, такими, как постоянные сверхтонкой структуры [18].

В настоящей работе на основе результатов расчетов иона $\mathrm{Nb}^{+3}$ и нейтрального атома $\mathrm{Nb}$ оценена погреш- 
ность в вычислении химического сдвига, возникающая из-за использования свойства пропорциональности валентных спиноров в остовной области атома $[5,16,17]$. Показано, что для химического сдвига линии $K_{\alpha 1}$ (переход $\left.2 p_{3 / 2} \rightarrow 1 s_{1 / 2}\right)$ использование свойства пропорциональности вносит погрешность около $20 \mathrm{meV}$, которая приемлема с точки зрения интерпретации эксперимента. Для химического сдвига линии $K_{\beta 1}$, соответствующей переходу из более диффузного одноэлектронного состояния $3 p_{3 / 2}$, учет поправок к свойству пропорциональности приводит к уменьшению погрешности расчета на порядок - со 100 до $10 \mathrm{meV}$.

Из анализа результатов расчетов химических сдвигов линий $K_{\alpha 1}$ и $K_{\beta 1}$ атома $\mathrm{Nb}$ в стехиометрических кластеpax $\left(\mathrm{Nb}_{2} \mathrm{O}_{5}\right)_{n}$ относительно кластеров $\mathrm{Nb}_{9}, \mathrm{Nb}_{16}$ следует, что существенное влияние на величину химического сдвига рентгеновских эмиссионных спектров атома в этих соединениях оказывает только его ближайшее окружение.

Исследование выполнено за счет гранта Российского научного фонда (проект № 14-31-00022). Расчеты проведены с использованием оборудования центра коллективного пользования „Комплекс моделирования и обработки данных исследовательских установок мегакласса“ НИЦ „Курчатовский институт“.

Авторы благодарны проф. К. ван Вюллену за предоставленную программу моделирования электронной структуры при помощи двухкомпонентного варианта теории функционала плотности [10]. Также авторы благодарят рецензента за полезные замечания.

\section{Список литературы}

[1] Joseph B. // Am. Mineral. 1955. V. 40. P. 805-827.

[2] Nakai I., Akimoto J., Imafuku M. et al. // Phys. and Chem. of Minerals. 1987. V. 15. N. 2. P. 113-124.

[3] Сумбаев О.И. // УФН. 1978. V. 124. № 2. Р. 281-306.

[4] Lomachuk Y.V., Titov A.V. // Phys. Rev. A. 2013. V. 88. P. 062511

[5] Titov A.V., Lomachuk Y.V., Skripnikov L.V. // Phys. Rev. A. 2014. V. 90. P. 052522

[6] Lomachuk Y.V., Maltsev D.A., Demidov Y.A. et al. // Nonlinear Phenomena in Complex Systems. 2017. V. 20. P. 170-176.

[7] Gatehouse B.M., Wadsley A.D. // Acta Crystallographica. 1964. V. 17. N. 12. P. $1545-1554$.

[8] Zhai H.-J., Döbler J., Sauer J., Wang L.-S. // J. American Chemical Society. 2007. V. 129. N. 43. P. 13270-13276.

[9] Mann J.E., Waller S.E., Rothgeb D.W., Jarrold C.C. // J. Chemical Physics. 2011. V. 135. N. 10. P. 104317.

[10] van Wüllen C. // Z. Phys. Chem. 2010. V. 224. P. 413-426.

[11] Titov A.V., Mosyagin N.S. // Int. J. Quantum Chem. 1999. V. 71. N. 5. P. 359-401.

[12] Mosyagin N.S., Zaitsevskii A.V., Titov A.V. // Review of Atomic and Molecular Physics. 2010. V. 1. N. 1. P. 63-72.

[13] Mosyagin N.S., Zaitsevskii A.V., Skripnikov L.V., Titov A.V. // Int. J. Quantum Chem. 2016. V. 116. N. 4. P. 301-315.
[14] Adamo C., Barone V. // J. Chem. Phys. 1999. V. 110. N. 13. P. 6158-6170.

[15] Schäfer A., Huber C., Ahlrichs R. // J. of Chemical Physics. 1994. V. 100. N. 8. P. 5829-5835.

[16] Titov A.V., Mosyagin N.S., Petrov A.N. et al. // Progr. Theor. Chem. Phys. 2006. V. 15. P. 253-283.

[17] Titov A.V., Mosyagin N.S., Petrov A.N., Isaev T.A. // Int. J. Quantum Chem. 2005. V. 104. N. 2. P. 223-239.

[18] Skripnikov L.V., Titov A.V. // Phys. Rev. A. 2015. V. 91. P. 042504.

[19] Skripnikov L.V., Petrov A.N., Mosyagin N.S. et al. // Phys. Rev. A. 2015. V. 92. P. 012521.

[20] Skripnikov L.V., Kudashov A.D., Petrov A.N., Titov A.V. // Phys. Rev. A. 2014. V. 90. P. 064501.

[21] Lee J., Chen J., Skripnikov L.V. et al. // Phys. Rev. A. 2013. V. 87. P. 022516.

[22] Skripnikov L.V. // J. Chem. Phys. 2016. V. 145. N. 21. P. 214301.

[23] Братцев В.Ф., Дейнека Г.Б., Тупищын И.И. // Изв. АН СССР. Сер. физ. 1977. Т. 41. №. 12. Р. 173-182.

[24] Майер И. Избранные главы квантовой химии: доказательства теорем и вывод формул. М.: БИНОМ. Лаборатория знаний, 2006. С. 197-267.

[25] Mosyagin N.S., Titov A.V.. Generalized relativistic effective core potentials. [Электронный pecypc.] Режим доступа: http://www.qchem.pnpi.spb.ru/recp.

[26] WebElements Periodic Table of the Elements. [Электронный ресурс.] Режим доступа: https://www.webelements.com/niobium/crystal_structure.html

[27] Sanville E., Kenny S.D., Smith R., Henkelman G. // J. Comput. Chem. 2007. V. 28. N. 5. P. 899-908.

[28] Tang W., Sanville E., Henkelman G. // J. Phys.: Condens. Matter. 2009. V. 21. N. 8. P. 084204. 